\title{
45 Years of Telescope Automation at MIT's Wallace Astrophysical Observatory
}

\author{
TC Brothers ${ }^{1 *}$
}

\begin{abstract}
Since the conception of the George R. Wallace Jr. Astrophysical Observatory (WAO) at MIT in 1971 we have had a focus on applying cutting-edge technology to astronomical equipment and enabling automated or remote observing for scientists and students alike. A key strength of our program has been including undergraduates in the research, design, and construction of systems. These experiences have led to breakthroughs that have been invaluable for both the observatory and the observers. Wallace Observatory was also the site of the first robotic telescope conference (1975), "Telescope Automation," which set stringent goals to which we compare our observatory today. Forty-five years later, looking back on the days of punch cards and tape reels at WAO, we must now ask, did we achieve the goals set out for us in the 1970's? Here, we discuss this question and examine our more recent efforts to automate our modern educational telescopes while looking forward to an exciting upgrade to one of our original telescope domes.
\end{abstract}

\section{Keywords}

Wallace Observatory; robotic telescopes; robotic observatories; astronomy education

${ }^{1}$ Massachusetts Institute of Technology

*Corresponding author: bro@mit.edu

\section{Introduction}

Since 1971, MIT's Wallace Astrophysical

Observatory (WAO) has made numerous advances in telescope robotics and automation while keeping a keen focus on education and the science of astronomy. Like any storied observatory, there were periods of significant advancement as well as setbacks. WAO was particularly successful in its early days, thanks to a fortunate paring of the explosive growth of computers in the 1970's and an intrepid team of scientists, engineers, and undergraduate students. Their laser focus on a robust suite of telescope automation designs, programs, and practices led to important contributions that still deserve recognition today. That said, it is equally important to recognize the automation advances by others that led to these breakthroughs.
More recently, the focus for WAO has been to fool-proof our robotic telescopes for remote use and embark on a journey toward fully automated and unattended observing. As we will see in next section, leaps in technology take time - in the case of WAO it took almost 4 decades to achieve this next goal!

\section{Early Telescope History}

While most readers are familiar with the contributions of Hans Lippershey (first terrestrial telescope - 1608) and Galileo Galilei (first astronomical telescope - 1609), most are likely unaware that it was Robert Hooke (Armitage, 1951) who designed the first sidereal rate telescope clock drive using a worm gear. Though the power sources have changed with the advent of electric motors, the mechanism for driving an equatorial telescope has changed little in over 300 years. With 
respect to imaging, the human eye was solely responsible for documenting observations until 1840, when an American, John William Draper, recorded the first astronomical image by taking a daguerreotype of the Moon (Abetti, 1954). The photographic technologies and methods of driving the telescopes progressed, but astronomy would remain a delightful, yet, labor intensive, tedious, and repetitive chore for the next 120 or so years.

There were various attempts to automate in creative ways in the 20th century (King, 1955), but none unlocked the accuracy and repeatability of computer-controlled telescopes (Bely, 2003).

\section{Wallace History}

The space race and computer age of the 1960 's-70's were a remarkable time for advancements in technology, and as a result, astronomy benefited immensely. In 1968, MIT began conceiving its own astronomical observatory (MIT, 1971). The captivated audience created by the NASA Apollo program resulted in MIT students' interest in astronomy-related courses increasing from 8 students per year to 500 (MIT, 1971) and yet, the university had no observatory. Further, an MIT research group had to schedule 18 months ahead of time for 70 nights of observation at other institutions annually (MIT-News, 1971). Something had to be done.

In 1971, a generous gift from MIT alumnus George R. Wallace, Jr., allowed the creation of a new observatory, 45 miles northwest of the MIT campus, far away from the Cambridge, MA city lights. An unoccupied hilltop was selected on an MIT-owned 1,300 acre rural property which was already home to the Millstone Radar and the 37-meter Haystack Radio Observatory, from which simultaneous optical/radio observations were envisioned. The location was a perfect balance of the dark skies necessary for optical observations and a location sufficiently close to campus that students could be transported to and from the site on a given night. It took approximately 2 years to clear the forested site, construct the main building and install the domes and telescope equipment (Figure 1, 2) (MIT, 1971).

The George R. Wallace Jr. Astrophysical Observatory (WAO) was built with an eye on education as well as student research and as initial Director Thomas B. McCord stated, “... a test site for new instrument development,” (MIT, 1971). From that vision, two telescopes were designed and built: a 16-inch Cassegrain for education and a 24-inch Cassegrain for planetary research and to serve as a testbed for astronomy technology. Both telescopes were mounted on German equatorial mounts and were built in Cambridge, MA by the Ealing Corporation. They were housed in 16-foot and 20 -foot domes, respectively, made by ObservaDome Inc.

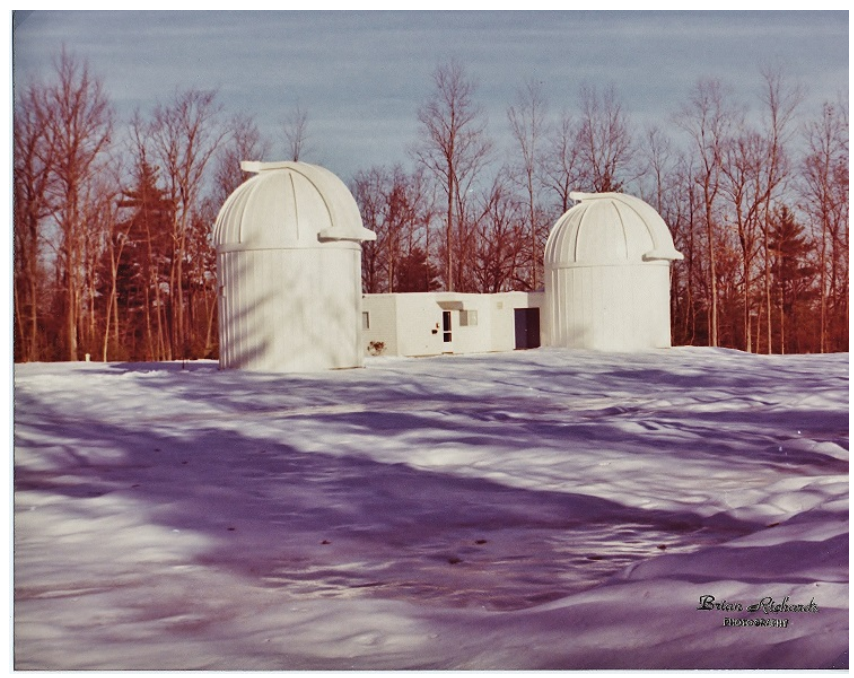

Figure 1. Wallace Observatory in winter. This photo shows the typical conditions in December through February, which happen to coincide with our best photometric nights. Photo (early 1970's) by Brian Richards.

\section{The MIT Automated Telescope}

From the outset, Director McCord hoped computers would eliminate the tedium of manual observing and free the observer from spending much of their valuable research time finding their objects. Though, looking back, McCord says,

"We were not yet pursuing a truly automated observatory for which no 


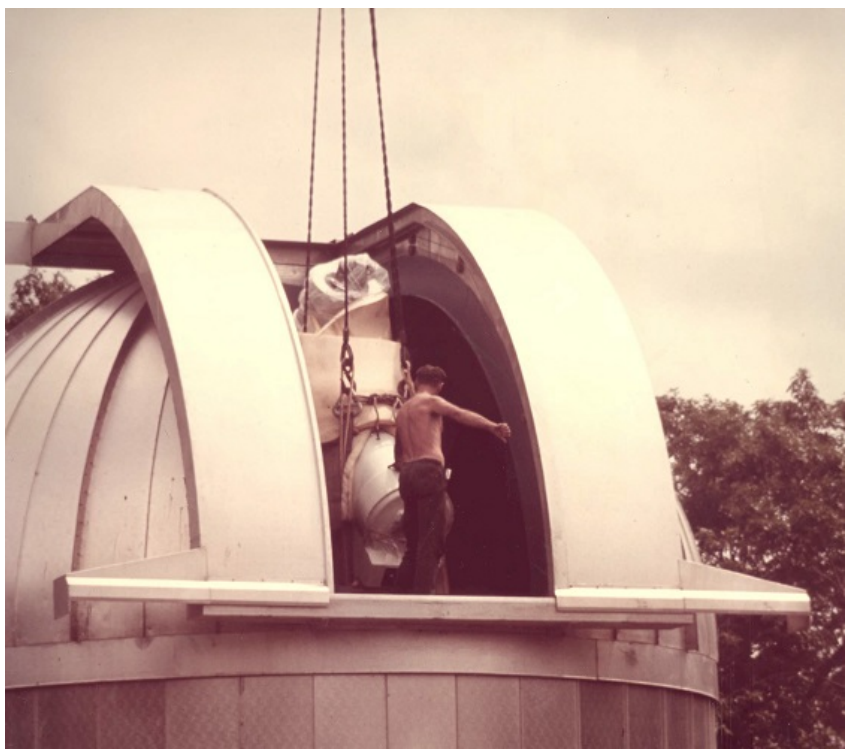

Figure 2. 16-inch telescope installation. Here we see, during installation in 1971, a crane guiding the Ealing 16-inch mount into place where it would live for 46 years.

one was to be present. We actually wanted to be at the telescope! We wanted the telescope to run mostly by itself and do complicated things. We also like to experiment with the latest electronic technology," (McCord, 2017).

Today, we would likely refer to this vision as a robotic observatory, in contrast to an automated observatory which refers to an unattended automated system.

A foundation of astronomy hardware and software programs had to be developed. McCord's thoughts prior to the dedication of WAO in 1971 were quite forward thinking, with plans to, "... program the telescope computer so that it will be familiar with star patterns and star brightness levels...", type an object name and slew to the desired target (aka "GoTo") within "a second of arc" and even compensate pointing for atmospheric refraction (Tech, 1971). These tasks we may take for granted decades later, but given that CCD cameras were invented in 1969 and computers only shortly earlier, it is clear that Wallace's early history was ambitious.
The first computer controlled, or robotic, telescope was operating reliably by the mid-1960s at the University of Wisconsin's Pine Bluff Observatory (McNall et al. 1968, Genet 2011). Shortly thereafter, there were several parallel projects to computerize telescope observations around the globe (McCord et al., 1975). Even so, the capability of MIT's automated 24-inch telescope and the breadth of its applications were remarkable for the time. Critical to these breakthroughs was a talented technical staff led by chief hardware engineer Grant Snellen, as seen in Figure 4. He was supported by Stephen Paavola, an adept software engineer, as well as by several undergraduates that were given the freedom to develop creative solutions to the automated observing problem under director McCord's leadership.

The core components of the 24 inch telescope's automation system included: a 24-bit Datacraft $6024 / 3$ computer to handle the command and control using FORTRAN programs, a Data General NOVA 1200 computer which served primarily as a pulse generator for the telescope drive's stepper motors, a storage tube Computek CRT display coupled with a graphics tablet and pen as a user interface, a Documation 1000 CPM reader and a Potter chain printer for entering programs into the system, two Cipher 9 channel 800BPI Magnetic Tape Drives, a Bryant memory drum to store the OS and serve as user scratch space, and two Century Data 111 disk drives (McCord et al., 1975). Much of the completed system is shown in Figure 3. By the dedication in 1971, much of the basic computer control was in place and operational.

In 1975, the MIT Wallace group put on the world's first robotic telescope conference, "Telescope Automation," and held it at MIT with over 40 professional astronomers and engineers from several countries in attendance (McCord et al., 1975). The topics ranged from how to use these new computers to analyze data in real time to the best way to operate radio and optical telescopes remotely and automatically.

The early achievements are almost too numerous to list but a few individuals stand out. An 


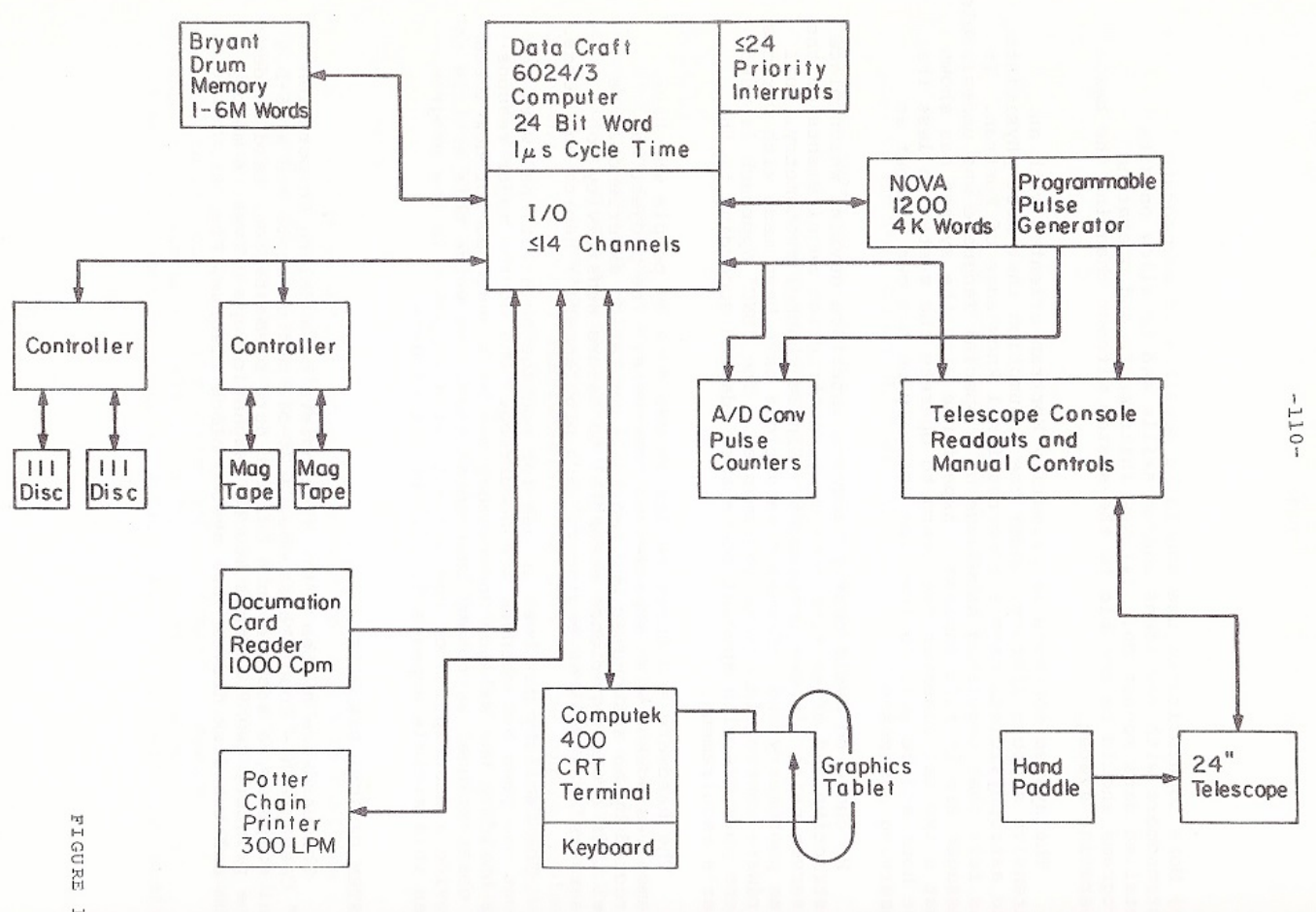

Figure 3. 24-inch control system. The guts of the MIT Automated Telescope, the computer system, user interface, associated equipment control and data recording devices.

undergraduate named Jim Gettys wrote much of the FORTRAN code for the screen editor which utilized an innovative user interface that featured a light pen and tablet where the user could select common functions such as "SLEW" or "ABORT, as well as more sophisticated operations such as "DUALCL" (an automated dual beam 25-filter chopping photometer control and data collection program) (Gettys 1975, Gettys and Schild 1977). Gettys also wrote enhancements to the slew program, including:

1. source coordinates from the Yale Bright Star Catalog where one could type, for example, "Alpha Lyrae" on the screen and the telescope would slew to the named star,

2. airmass display for planning observations,

3. and a raster scan program that would move the telescope back and forth over an area of sky.
Observatory engineer, Michael Brookes, was instrumental in many of the hardware applications. In particular, he designed and built a novel and inexpensive dome automation system using toy magnets (Brookes, 1974).

Also impressive was the pointing accuracy of the finished, computer-controlled 24-inch telescope, which is recorded as 10 arcseconds in an early Wallace logbook. Even today, with a modern control system, we still see 10 arcseconds RMS pointing on this very same telescope.

By all accounts from published papers, proceedings, and personal interviews, it is quite clear that McCord's dream became a reality. The innovative team of professors, engineers and undergraduates had made an important contribution to the field of astronomy technology. Within two years they had indeed created an automated observatory from scratch at a time when the path was uncharted (McCord et al., 1975). Even by today's standards, their achievement in such a short period of time is impressive. 


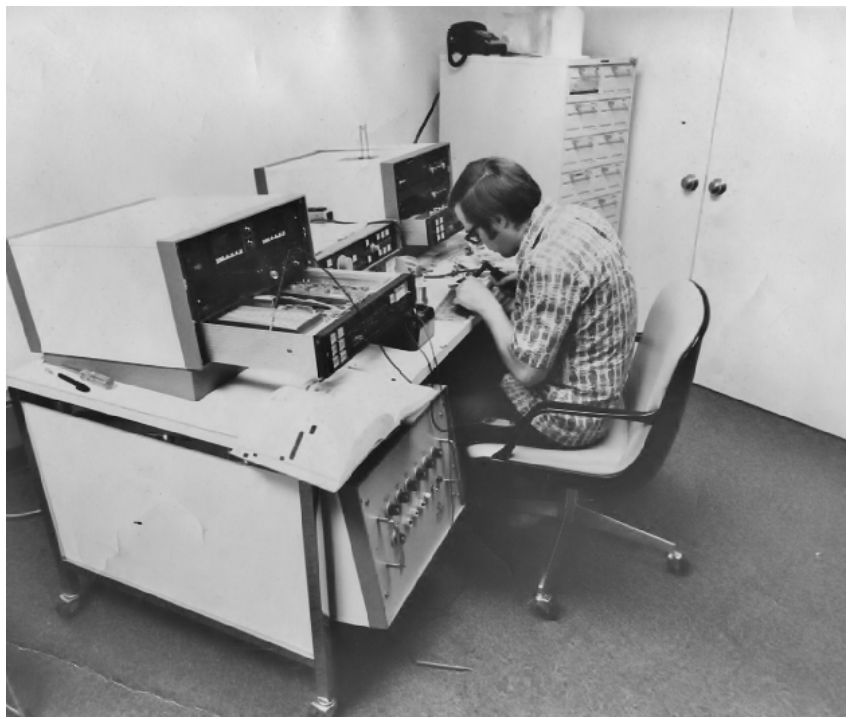

Figure 4. Grant Snellen, engineer, seen here developing the 24 inch telescope control system in 1971. Given the size of 70s-era computer systems, it occupied nearly $50 \%$ of the observatory building footprint.

After several years of achievement (McCord 2017, McCord et al. 1975, Gettys 2017) the original team moved on to new things and the system became difficult to maintain, at which point a new drive system was installed. Somewhere along the way the Brookes dome automation system was disassembled and the dome returned to its original state of manual operation for the next few decades. Even today, careful inspection reveals a few button magnets hidden around the dome sill - a reminder of the ingenuity of that era.

\section{Robotic, Remote}

In 1983, then director James Elliot, devised a plan to enhance the offerings for undergraduate students in his introductory astronomy classes (MIT-Office of the President, 1984). A roll-off-roof observatory, affectionately referred to as "the Shed" by students, was constructed with 4 isolated concrete piers that were driven down to bedrock. Celestron C14, 14-inch, "orange tube" Schmidt-Cassegrain telescopes mounted on equatorial fork mounts, driven by Byers Retrofit Starmaster clock drives, occupied each pier by 1984. Students would travel out with Prof. Elliot or a teaching assistant and star

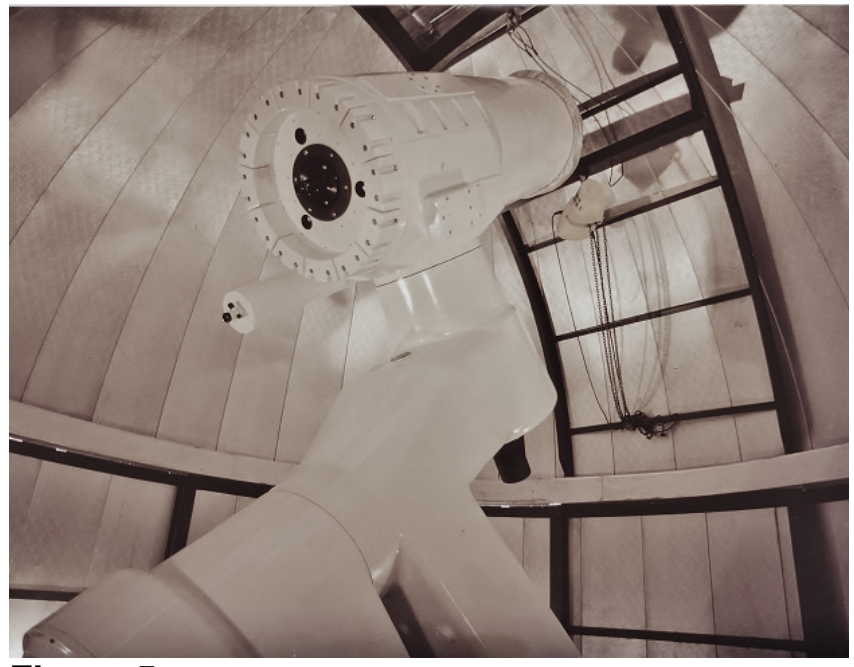

Figure 5. The MIT Automated Telescope. The monstrous 24-inch telescope is seen here in its original configuration, pointing towards the celestial pole during testing.

hop to their targets with the aid of setting circles, finder charts, and Telrads.

Elliot also had a strong desire to make Wallace telescopes remotely accessible via various forms of communications technology of the time, including modems over telephone lines, direct microwave links to campus, and later, over a T1 link. (Leiner, 1989) The Telescience Testbed Pilot Program, funded by NASA, was meant to develop the remote control capabilities of science experiments that might relate to the planned International Space Station. WAO was selected as one of several testbeds to experiment with such technology (Ritterbuch, 1987). While communication was possible, bandwidth limitations for in-situ visualization, transferring data, and data storage showed the need for more robust internet infrastructure for such tasks.

Another limitation was the observatory itself. The Shed roof, as well as the 24 and 16-inch domes, were manually operated. The Shed roof and dome rotation required local manipulation of the controls, and the dome slits required use of a hand crank. To make matters worse, in a typical New England winter, the Shed roof friction drive wheels would make poor contact with the rails due to thermal contraction. In the event the roof was stuck open or 
closed during classes, a rope was tied to the rafters and some students would pull while a TA would push the drive button to attempt to move the 3,000 lbs. roof. This was obviously not an optimal procedure from the standpoint of reliable observing or safety.

A third limitation was that "GoTo" telescopes were commercially available by the 1990's but were not reliable or precise enough for remote use. Much of the peripheral technology (focusers, filter wheels, detectors, spectrographs, and such) were not well integrated with astronomy software quite yet.

In the early 2000's, more robust and reliable astronomy equipment and software became commercially available for the predominant operating system, Microsoft Windows. The ASCOM platform (Astronomy Common Object Model) was released for Windows machines in 2001, which widened the net for more astronomy related instruments to be tied together in automated sequences (The ASCOM Initiative, 2018).

Software Bisque released the Paramount ME robotic German equatorial mount in 2002 (Bisque, $2018 b$ ). This versatile telescope mount could handle a variety of instrument packages up to 150 lbs. (Bisque, 2018a) and coupled with Bisque's TheSky observatory control software, resulted in a fully functional robotic observing package.

Several key improvements to the site and the equipment in the observatory paved the way to reliable remote observation at WAO during the short but highly effective tenure of Alan Midkiff as observatory manager until 2009, when the author assumed this role. The network had recently been overhauled to include a fiber optic link directly to campus and was sequentially upgraded from $1 \mathrm{Mbs}$ to 10 to 100 , and by 2010 was operating at $1 \mathrm{Gbs}$. In 2006 and 2008, two Paramount ME's were purchased, as well as a complement of four identical instrument systems for the shed telescopes. Each instrument consisted of an SBIG STL-1001e CCD with integrated eight-position filter wheel holding Custom Scientific Inc. 2 inch Johnson/Cousins filter set and Optec TCF-S3 electronic focuser. These two robotic mounts replaced two of the Celestron manual mounts while preserving all 4 of the C-14's. This meant that during classes, all students in the Shed would effectively have identical optical capabilities, while more experienced students and staff would have access to the two remote-capable Paramount ME's via Microsoft's Remote Desktop client.

For the next two years, remote use was dictated by the availability of the observatory site manager to be able to physically monitor the weather, make the decision to open the Shed roof, communicate with the campus-based operator by phone, reboot computers and equipment as needed, make emergency close decisions if weather soured, and then stand by in case things improved. While this worked for the students if schedules aligned, it was not an efficient use of observatory staff time. Bisque's Orchestrate scripting software was experimented with in those years in an attempt to eliminate some of this tedium. Providing the roof was manually opened, the software could automate an imaging run, but it could not make it fully autonomous due to a lack of feedback from parameters such as weather. Despite this, there were numerous successful undergraduate projects resulting from this era, and feedback from students informed staff of what they should strive for next.

In 2011, we lost our beloved director Prof. Elliot, but we did not lose our way. Associate Director Dr. Michael Person assumed the Director position and we continued our path towards more effective robotic observing.

\section{Remote Automation Achieved}

Director Person sought to make the next step forward in remote observing at WAO to solve some very real-world observational problems. The drive time for commuting to and from the observatory was increasing (often 90+ minutes each way during rush hours) and students required more than a handful of data points at the cost of several hours per night to complete a typical class or thesis project, whether it be a photometric light curve of an asteroid, an exoplanet transit or spectra from a binary star system. Person stated, 
"This meant they needed to be able to take data all night, as the driving commitment made short trips very wasteful, as well as interfering with their other academic commitments. If commuting could be eliminated, they could take data in short bursts, preferably from the comforts of campus, after they've been initially trained to observe on site."

(Person, 2017)

The observatory is also subject to the infamous New England weather. As Mark Twain once said, "If you don't like the weather in New England now, just wait a few minutes." This meant it was not uncommon for a class outing to be ruined by unexpected clouds. The unpredictable microclimate around the observatory, the wide yearly temperature range $\left(-20^{\circ} \mathrm{F}\right.$ to $\left.110^{\circ} \mathrm{F}\right)$ and the unstable grid power meant there needed to be a reliable weather trigger and mechanism for shutting operations down to protect the equipment in the event of an emergency.

By 2013, plans were well underway to make remote robotic use reliable. The most logical place to start was the Shed, given that it contained two robotic telescope systems and much of the hiccups related to remote use had been worked out in years prior. The plan was threefold:

1. rearrange the telescopes so that the robotic mounts enjoyed the most unobstructed observing time, meaning they would be mounted on the middle two piers,

2. make the roll-off roof reliable and ASCOM-based so any scripting software could address it,

3. and create a sensor suite that would give the remote observer an "almost there" feeling from the terminal and add safeguards to the equipment during automation.

In the winter of 2013 a 15-foot tower was erected near the northeast corner of the main building. On the tower the following sensors were attached:
- a Masterclock NTP100-GPS GPS antenna and NTP server to keep precise time within 2 ms on all observatory machines,

- Diffraction Limited Boltwood II Cloud Sensor for issuing open/close conditions to the automation hardware and software,

- Unihedron SQM-LE to measure a calibrated magnitude per arcsecond ${ }^{2}$ in a $20^{\circ}$ FWHM cone around zenith,

- and an SBIG color All-Sky Camera to create a $180^{\circ}$ visual record of the current sky conditions as well as a time-lapse of the previous 12 hours.

In addition, we purchased an updated Davis Vantage Pro 2 weather logger for more accurate weather info which uploads measurements constantly to Weatherunderground.com to assist in local weather predictions. Trendnet PTZ (pan, tilt, zoom) IP cameras were installed throughout the observatory and were made viewable via the weather computer or any web browser remotely. The cameras have an IR LED for night vision and microphones so the user can hear even the faintest beeping alert code from various pieces of hardware. Reflective stickers (McMaster \#6042T11) are used at the open and closed positions of the roof to verify remote operations.

A few weeks later PierTech, Inc. installed a control retrofit system in the Shed. One of the friction drives was removed and replaced with a cogwheel driving a track permanently mounted to the rail. The AC motor was replaced with a variable frequency drive to enable control of the roof speed and limit switches to limit travel. On the control box there are also inputs for emergency weather closure and an interrupt for park sensors. Due to the continued local use of the Shed for class instruction, it did not make sense to use the hardware-based weather closure or we could potentially close the observatory roof on the manually operated telescopes at either end of the building. On the other hand, the park sensor input 
proved invaluable for preventing disasters such as accidentally closing the roof on a telescope pointing at the sky. EATON 1450E-6514 non-polarized photoelectric sensors were connected in series to verify the two robotic mounts were within $1^{\circ}$ of park position, due South and $10^{\circ}$ elevation. To control the PierTech roof system locally the user simply presses appropriate buttons on the touchscreen.

For remote use, we employed a B\&B Electronics VESP211-232 virtual COM port server. This device was given a permanent IP address that any observatory computer could assign as a virtual COM port. This solved the problem of controlling the roof remotely and not having to create a messy solution to physically serve a serial connection. Occasionally, as with any serial connection, handshaking can go wrong and the device can hang. This applies to many pieces of astronomy equipment that still rely on serial to USB connections for communication.

The solution was to use an addressable Web Power Switch 7 from Digital Loggers where each outlet can be individually cycled and can even be scripted now via the ASCOM interface. The ability to control outlets remotely and systematically has salvaged countless observing sessions.

By the nature of where observatory locations are often chosen, far from civilization, power can often be suspect. This is even true 40 miles from Boston. Uninterrupted power supplies (UPS) are an essential component to reliable observing. In the case of the roof controller we chose a UPS that had the capacity to operate the roof at least twice in the event of power failure in the winter. The reasoning behind that level of specificity is due to the fact that lead acid batteries found in such devices begin losing capacity as temperature falls, potentially near $50 \%$ by $0^{\circ} \mathrm{F}$ (Hutchinson, 2004). For this reason an APC Smart-UPS 1000 was chosen. Another complication is GFCI outlets, often required by code for occupied outdoor buildings, which will immediately be tripped by plugging in a typical UPS., as outlet may sense leakage current to ground on the circuit. The solution is to choose a
UPS that has UL code 1778 in its specifications. By the fall of 2014, the two robotic Shed telescopes were being used regularly by classes for remote observing sessions run by Person from a designated lab on campus. The current Shed configuration can be seen in Figure 6 .

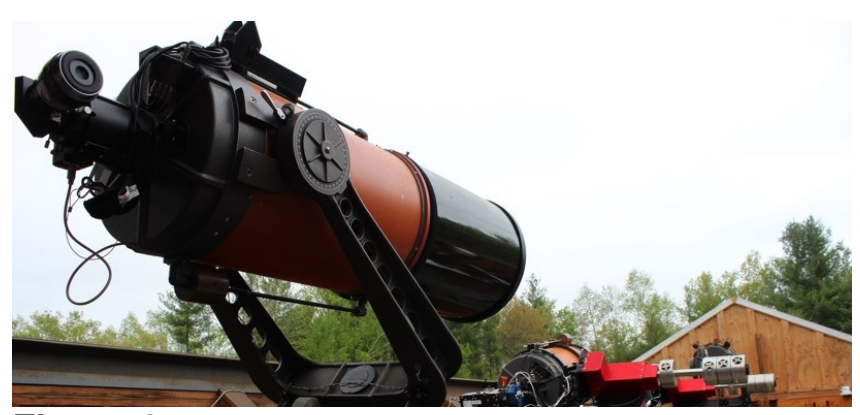

Figure 6. Today the WAO Shed consists of four refurbished 14-inch telescopes, two of which are on manual mounts and two are on Bisque Paramount ME's.

\section{Unattended Automation}

During the first year of remote use, the author began experimenting with full automation of one of the Paramount ME's in the Shed. There were a few choices of scripting/scheduling programs on the market to initiate the automation. While most dedicated robotic observatories use ACP by DC-3 Dreams, with over 800 reported installations, it was not possible for us at the time due to funding constraints. With the complicated nature of a mixed mode system requiring both local and remote control, we decided to first try the license we already owned, CCDWare's CCDAutoPilot. Although the scripting software seems mostly focused on astrophotography, we did find it to be a very acceptable solution for individual projects. In addition to all of the expected features, such as slewing, imaging, focusing, automated calibration runs, etc., it has several nice features that take into account our tower sensors. It will embed into each FITS image header the weather data from either of our weather sensors as well as night sky brightness measurements from our sky quality meter. These have proven invaluable when combined with the all sky imager's recordings when trying to piece 
together spurious changes in image quality or background intensity. A classic example is when a security guard approaches the building with high-beams on during operations. The data point can be safely eliminated and further confusion avoided.

Testing of the system as a whole occurred in two stages. First was an automated local observation where the author was present in the main control room and monitored the scripted observation on the Shed telescope on one screen and the observatory control computer on another screen, as seen in Figure 7. The goal was to take several guided 10-minute images in different filters of Messier 110. The first attempt was likely too ambitious and resulted in several focusing failures. The author was able to monitor the log, interrupt the process and resume the task with success.

The second test was an automated remote session, occurring one week later in November 2014. The author crafted a mosaic in theSkyX software, a $5 \mathrm{x}$ 5 grid, to be repeated 5 times for the purpose of detecting minor planets (Figure 7). The experience from the previous attempt was taken into account and focusing routines were adjusted to avoid focusing on very bright stars off field, and instead to find the brightest star in any particular mosaic field once every hour. This saved a great deal of time by minimizing the number of slews and settling time. After the program was scheduled, the run button was pressed and the author went home for dinner, taking care not to peak at the current status of the operation until it was well underway. The operation was checked once, a few hours into the plan and the author was able to get a full night's rest and awoke the next morning to 125 data frames as expected with only one unusable frame. Adequate sleep enabled timely reduction and analysis by 10AM, particularly important if a near-Earth asteroid is to be discovered. While not every scientific observation can be trusted to automation, simple searches and photometry/astrometry projects seem to be most suitable for automated observing.

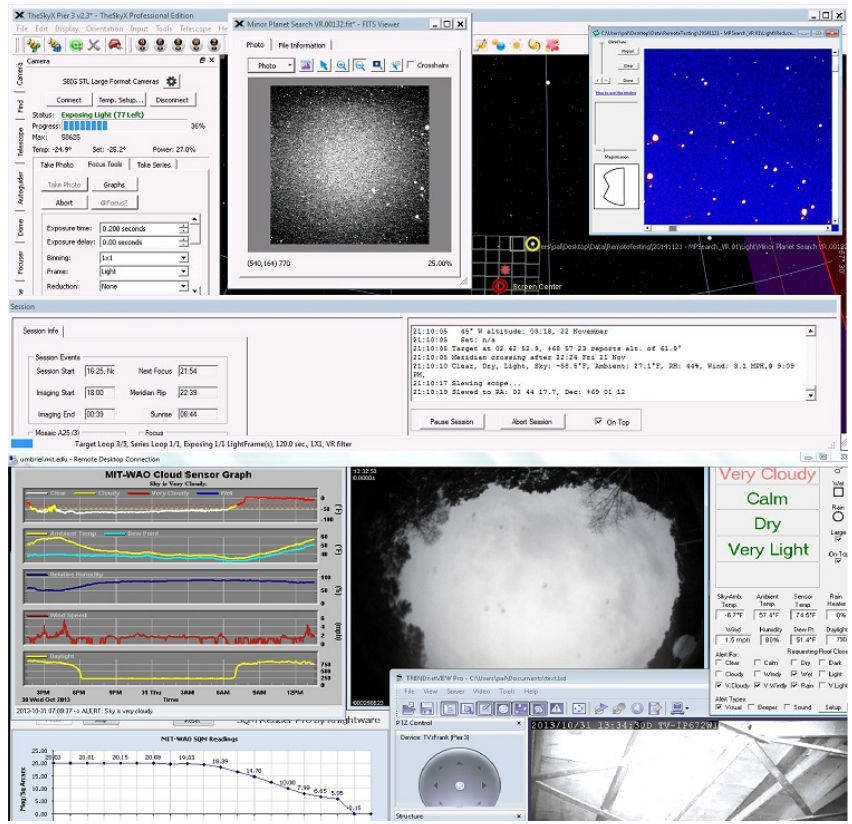

Figure 7. The remote observer's viewpoint. The above images show the necessity of two monitors to display all the necessary info to give the "almost there" feeling to operators. The second image shows the "first light" of our recent automation efforts, where CCDAutoPilot runs a $5 \times 5$ asteroid search grid, repeated 5 times over 1 night. We also see the program PinPoint from DC-3 Dreams which is used for astrometry and asteroid detection.

\section{SAURON}

Although extremely successful, the automation in the shed presented a new problem. If the observatory is mixed use, how must safety procedures be crafted to avoid possible mishaps if local users are present operating one telescope while a remote user is off-site running a scripted observation? The Shed automation system is still available and serves as an automation testbed, but it has received sporadic use in that mode given the numerous conflicts with classes needing the equipment on those valuable clear nights. So, we embarked upon a parallel approach with a new isolated dome.

The project was intended from the start to be a rapid prototype for a possible larger telescope system down the road. We used a leftover pad and 
pier that have been undeveloped since 1972. A 10-foot Technical Innovations dome was installed by the author and several undergraduate researchers over the course of the summer months of 2015. The students gained valuable observatory construction experience and learned how to use a large variety of power tools. Inside the dome, we used a new Paramount ME II mount and a spare Celestron C-11 11-inch telescope and an older SBIG ST-7XME CCD for testing. Undergraduate student Molly Kosiarek used the system to write her senior thesis, which resulted in a peer-review publication where the project was named, SAURON (Kosiarek et al., 2017).

While the mount, telescope, and detectors all worked as expected, the dome was never reliable. There were numerous shortcomings in the mechanical design that had to be re-engineered and the control system was unreliable for automation purposes. It is still actively used by undergraduates as a testbed for their robotic observing ideas.

\section{Old Telescopes Revisited}

Amidst all of these experiences over the past decade, the two original telescopes remained in various states of operation. The 24 -inch has gone through a few more control system retrofits. For the better part of a decade we ran the 24-inch on Lowell Observatory's MOVE system, developed by Larry Wasserman. Over time it became difficult to maintain given its reliance on an unsupported control system and the need to run it on a Windows 98 machine with heavy use of analog logic circuits. This is a good example of why a telescope system should be independent of any one particular person or OS. We experimented with a Chronos retrofit system but deemed it to be incompatible with our telescope. Finally we settled on the Sidereal Technology ForceOne brushless servo motor controller which required very little modification of the telescope drive system and its limit and home switches and encoders. We went through several revisions of this system as it progressed from prototype to product, and today it is used in many commercially available telescope systems.
While the control system revived an otherwise dead telescope, the telescope itself is approaching 46 years of age with a design that is even older, making it a reasonable candidate for remote use if we automated the dome controls, but not a reliable candidate for autonomous use.

The 16-inch system had numerous down times throughout its life and suffered from poor design and lack of maintenance. In 2008, it was retrofitted with ComSoft's stepper motor system, and in 2013 it began having significant mechanical issues resulting in a stuck RA worm assembly. Here too we applied a Sidereal Technology system of the brushed DC motor variety - the SiTech Servo II controller. That summer a group of summer students worked tirelessly to breathe new life into the system and it worked fairly well for another 3 years. By fall 2016 it began requiring nightly support calls and it was decided to turn the ailing telescope into an opportunity to re-imagine the dome.

\section{The Future of WAO Robotic Observing}

In 2017, a consortium of MIT departments and alumni funded our proposal to decommission the MIT 16-inch telescope and completely upgrade the telescope and dome system (Table 1). The dome was retrofitted with a custom automation system by Greg Stafford of Observatory Automation Solutions and uses a Diffraction Limited MaxDome II controller with ASCOM drivers. The dome was painted inside and out, a new rubber flooring was added for safety, new ventilation fans were installed to increase ambient air flow, and the pier was restored to its original condition. It was determined that the optimal telescope to fit in the dome was a 24-inch Planewave CDK. The fused silica option was selected to minimize thermal expansion, refocusing time, and out of focus travel, which were problems with the original Ealing telescopes. The mount is a Software Bisque Taurus 600 equatorial fork mount. This mount was selected for several reasons, including: the stability of a fork design, the elimination of meridian 
flipping which had plagued us with pointing and optical issues in our German equatorial mounts, and our deep understanding of the Bisque TCS system, making troubleshooting and management much less taxing. It also has Renishaw absolute axis encoders to negate the need to autoguide for most projects and a special firmware package to enhance satellite tracking capabilities. This is especially important given the recent uptick in requests from MIT's own AeroAstro department.

Table 1 Taurus and Ealing 24-inch comparison.

\begin{tabular}{|l|l|l|}
\hline & $\begin{array}{l}\text { Taurus 600 } \\
\text { (Predicted) }\end{array}$ & $\begin{array}{l}\text { 16-inch Ealing } \\
\text { w/SiTech Retrofit } \\
\text { (Measured) }\end{array}$ \\
\hline $\begin{array}{l}\text { pointing } \\
\text { accuracy } \\
\text { (all sky model) }\end{array}$ & $\begin{array}{l}10 \text { arcsecond } \\
\text { RMS }\end{array}$ & $\begin{array}{l}\sim 90 \\
\text { arcseconds }\end{array}$ \\
\hline $\begin{array}{l}\text { theoretical } \\
\text { pointing } \\
\text { precision }\end{array}$ & 0.1 arcsecond & $\begin{array}{l}16.6 \\
\text { arcseconds }\end{array}$ \\
\hline slew speed & 2.5 degrees/s & 1 degree/s \\
\hline $\begin{array}{l}\text { tracking } \\
\text { accuracy over } 10 \\
\text { minutes }\end{array}$ & 1 arcsecond & $\begin{array}{l}15-20 \\
\text { arcseconds }\end{array}$ \\
\hline
\end{tabular}

On the detector side of the system we are using an Optec Perseus 4-port instrument selector to add a great deal of versatility to the system. We had been using an earlier version of this device for many years on the Ealing 24-inch and are happy to report it has enabled us to quickly and seamlessly switch between wide field imaging, high speed imaging, spectroscopy and an eyepiece for educational outreach while maintaining collimation and nearly parfocal spacing for each port. We currently use each port as follows,

1. 16 megapixel FLI 16803 wide field imaging CCD with Sloan filters for photometry/astrometry projects,

2. 2-inch eyepiece for tours and outreach,

3. high-speed GPS-timed camera for recording occultations and exoplanet transits,
4. and spectrograph (Table 2).

FLI CCD is a good match with the Planewave optics and produces a field 2.5 times larger than that of the Ealing 24-inch, while the increase in aperture collects 2.25 times more light than the Ealing 16-inch it replaces. The computer is a Nuvo-5006LP rugged computer designed to handle temperatures from $-13^{\circ} \mathrm{F}$ to $158^{\circ} \mathrm{F}$.

Table 2 New 24-inch Instrument Payload

\begin{tabular}{|l|l|l|l|}
\hline $\begin{array}{l}\text { Port } \\
\#\end{array}$ & Instrument & Model & $\begin{array}{l}\text { Details / } \\
\text { Features }\end{array}$ \\
\hline 1 & CCD & FLI 16803 & Sloan filters \\
\hline 2 & CCD & $\begin{array}{l}\text { ZWO } \\
\text { ATI174mm- } \\
\text { Cooled }\end{array}$ & $\begin{array}{l}\text { High-speed } \\
\text { GPS-timed } \\
\text { camera for } \\
\text { recording } \\
\text { occultations } \\
\text { and exoplanet } \\
\text { transits }\end{array}$ \\
\hline 3 & $\begin{array}{l}\text { Spectrograph } \\
\text { /CCD }\end{array}$ & $\begin{array}{l}\text { Shelyak } \\
\text { Lhires III / } \\
\text { QSI 683 }\end{array}$ & $\begin{array}{l}\text { 5 gratings } \\
\text { swappable } \\
\text { gratings and } \\
\text { neon } \\
\text { calibration } \\
\text { lamp }\end{array}$ \\
\hline 4 & Eyepiece & $\begin{array}{l}\text { TeleVue } \\
55 \mathrm{~mm}\end{array}$ & $\begin{array}{l}0.7^{\circ} \text { field of } \\
\text { view }\end{array}$ \\
\hline
\end{tabular}

As of the writing of this paper the mount and telescope have been installed and we are currently waiting on the Perseus to achieve first light with final instruments, (Figure 8). Initial tests conducted with a mounted $50 \mathrm{~mm}$ Lunt solar telescope for the solar eclipse on August 21, 2017 demonstrated the mount's remarkable capability, even with an extremely rough polar alignment, to slew to our target, the Sun, track it for four hours, and conduct a live broadcast of the event. On August 30th, 2017 we conducted collimation and basic pointing tests using a USB camera. Using the drift polar alignment method, and 7 mapped points, we are already seeing pointing accuracy of 15 arcseconds RMS. We hope to have the system ready in the coming weeks to take spectrographic data for fall 
classes. While waiting for the new equipment to arrive this spring, an undergraduate conducted initial experiments on simultaneous photometric and spectroscopy measurements using the old Perseus and detectors mounted on Ealing 24-inch (Patra, 2017). We are very optimistic about similar experiments in the future on the new system.

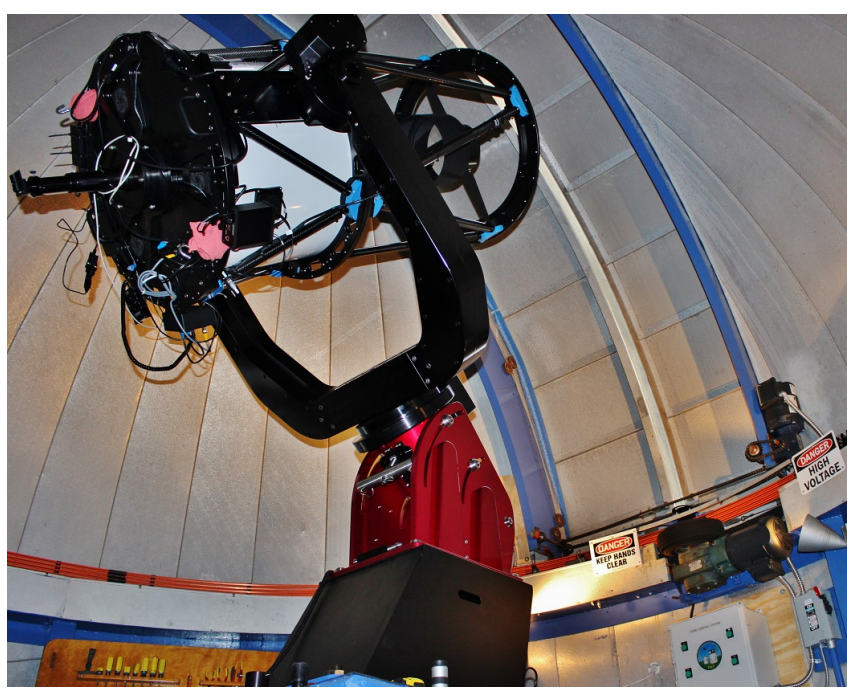

Figure 8. The new Wallace 24-inch telescope. The mount has performed as expected so far and we expect to have its full complement of instruments by the fall 2017 semester start.

\section{Conclusion}

From interviews conducted, whether it be the original occupants or the incoming freshman, the spirit of Wallace is still alive as our observatory continues to grow (Figure 9). Our observers still wish to push robotic observing forward, but there is still a deep desire to maintain a physical connection to the observatory. After initial testing of our new robotic 24-inch telescope, our plan is to use ACP to fully automate the dome on every night other than fall class nights. Students are already anxiously devising creative ways to use the telescope and all of its new capabilities.

\section{Acknowledgements}

The author would like to thank everyone who has put up with his ideas and have generously supported them, in no particular order: the late

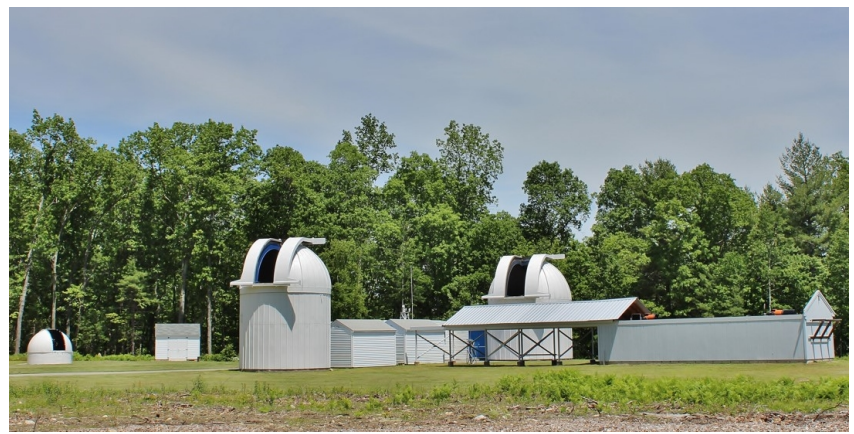

Figure 9. Wallace 2017. Today, MIT's Wallace Astrophysical Observatory consists of two 24-inch telescopes, four 14-inch, an 11-inch and a portable 14-inch. Of those, four are now available for remote robotic use from campus.

Prof. James Elliot, Dr. Michael Person, and my family who puts up with my crazy hours. Also, Tom McCord and Jim Gettys who have been very generous in their time to set the record straight.

\section{References}

Abetti, G. (1954). The history of astronomy. London: Sidgwick and Jackson, 1954, page 326.

Armitage, A. (1951). Robert Hooke as an astronomer. Popular Astronomy, 59:287-298.

Bely, P. Y. (2003). The Design and Construction of Large Optical Telescopes. The design and construction of large optical telescopes, by Pierre $Y$. Bely. Berlin, New York: Springer.

Bisque, S. (2018a). Paramount ME (archived page, out of production). http://www.bisque.com/ sc/pages/Paramount-ME.aspx Online; accessed 22/1/18

Bisque, S. (2018b). Software Bisque History and Milestones. http://www.bisque.com/sc/pages/ Timeline.aspx Online; accessed 22/1/18.

Brookes, M. (1974). An inexpensive automatic telescope dome controller. Review of Scientific Instruments, 45(11):1372-1374.

Genet, R. M. (2011). History of Robotic and Remotely Operated Telescopes: The Fairborn Ob- 
servatory 1979-1989. In Conference Proceedings from Telescopes from Afar.

Gettys, J. (1975). An Interactive Data Collection Program for Use with an Automated Photometer and Telescope. In Telescope Automation, page 179.

Gettys, J. (2017). Personal Communication, $8 / 2 / 2017$.

Gettys, J. and Schild, R. E. (1977). A Photometric Peculiarity Index in the AP Stars. Publications of the Astronomical Society of the Pacific, 89(530):519.

Hutchinson, R. (2004). Temperature effects on sealed lead acid batteries and charging techniques to prolong cycle life. Technical report, Sandia National Laboratories.

King, H. C. (1955). The history of the telescope. High Wycombe: Charles Griffin, pages 302, 330, 334, 392, 412.

Kosiarek, M., Mansfield, M., Brothers, T., Bates, H., Aviles, R., Brode-Roger, O., Person, M., and Russel, M. (2017). SAURON: The Wallace Observatory Small AUtonomous Robotic Optical Nightwatcher. Publications of the Astronomical Society of the Pacific, 129(977):075001.

Leiner, B. M. (1989). Telescience testbed pilot program, Final Report: Volume I, Executive Summary.

McCord, T. (2017). Personal Communication, 6/5/2017-8/2/2017.

McCord, T. B., Paavola, S. H., and Snellen, G. (1975). The MIT Automated Optical Telescope. In Telescope Automation, page 108. Proceedings of an International Conference held at MIT, Cambridge, MA USA, April 29 - May 1, 1975.

McNall, J. F., Miedaner, T. L., and Code, A. D. (1968). A computer-controlled photometric telescope. The Astronomical Journal, 73:756.
MIT (1971). Tech talk: Wallace Observatory Dedicated Last Week, 10/20/71.

MIT-News (1971). Wallace Observatory Dedication Ceremony, 10/14/71.

MIT-Office of the President (1984). Report of the President 1983-1984.

Patra, K. C. (2017). Optical method for mapping interstellar dust, 8.14 Junior Lab. Massachusetts Institute of Technology, Department of Physics.

Person, M. (2017). Personal Communication, $8 / 31 / 2017$.

Ritterbuch, S. (1987). Automating Wallace and Testing Telescience.

Tech, M.-T. (1971). MIT gains new "eyes" on the stars, 10/1/71.

The ASCOM Initiative (2018). History of ASCOM. http://ascom-standards.org/About/History. htm Online; accessed 22/1/18. 